\title{
Revealing the Therapeutic Targets and Mechanism of Baicalin for Anti-chronic Gastritis Through TMT Proteomic Approach
}

\section{Wanli Ji}

Shanghai University of Medicine and Health Sciences

\section{Yan Huo}

Shanghai University of TCM: Shanghai University of Traditional Chinese Medicine

\section{Yu Zhang}

Shanghai University of Medicine and Health Sciences

\section{Xinhong Wang}

Shanghai University of TCM: Shanghai University of Traditional Chinese Medicine

Yifan Zhang ( $\nabla$ yifan1210@163.com )

Shanghai University of Medicine and Health Sciences

\section{Research}

Keywords: baicalin, ethanol, chronic gastritis, quantitative proteomics, targets

Posted Date: October 12th, 2021

DOl: https://doi.org/10.21203/rs.3.rs-924045/v1

License: (c) (i) This work is licensed under a Creative Commons Attribution 4.0 International License. Read Full License 


\section{Abstract}

\section{Background}

Baicalin $(\mathrm{BCL})$ is a natural compound with beneficial activities, including antioxidant, anti-inflammatory and immunomodulatory. To investigate the therapeutic action of baicalin treatment in ethanol-induced chronic gastritis. Here, we investigated the proteome changes in the gastric tissue to elucidate the therapeutic targets of baicalin in chronic gastritis by TMT-based quantitative proteomics.

Results

Using TMT-based quantitative proteomics, a total of the 4,452 proteins were identified and quantified in the gastric antrum tissue of Sprague-Dawley rats. Of these, 107 differentially expressed proteins, including 44 up-regulated and 63 down-regulated proteins, were uncovered in the baicalin-treated group as compared with the untreated group with ethanol-induced gastritis. Furthermore, the expression of TPM2, GIMAP4, and Mpc1 was validated using Western Blot. Baicalin could decrease the production of interleukin (IL)-2, IL-8 and tumor necrosis factor-a (TNF-a), while increase the expression of epidermal growth factor (EGF) and B-cell lymphoma-2 (Bcl-2). Notably, protein-protein interaction network analysis revealed the widespread interactions mediated by baicalin.

\section{Conclusions}

We investigated the effects and potential mechanism of baicalin in chronic gastritis. Proteomic technology was used to explore baicalin-affected proteins and some signaling pathways. The results may provide important insights into the discovery of potential target proteins for the treatment of chronic gastritis.

\section{Introduction}

Long-term alcoholism may cause chronic gastritis, and approximately $10 \%$ of the world's population has gastritis or gastric ulcer [1]. Ethanol exerts direct effects on the digestive system and interferes with the digestive functions [2]. It was demonstrated that ethanol-induced chronic gastritis is highly likely to trigger gastric mucosal injury, owing to the high sensitivity of the stomach mucosal tissue, which is easily irritated by external factors. In particular, ethanol may severely corrode and destroy the physiological environment required for the normal function of the gastric mucosa $[3,4]$. Furthermore, the damage caused to the gastric mucosa is accelerated upon binding of acetaldehyde to gastric mucosal proteins [5]. High concentrations of ethanol ( $\geq 7 \% \mathrm{v} / \mathrm{v})$ exert a wide variety of toxic effects, including alteration in both passive and $\mathrm{H}^{+}-\mathrm{K}^{+}-\mathrm{ATPase}$ dependent transport of protons across cell membranes [6].

At present, the treatment of gastritis is designed with the aim to reduce the secretion of gastric acid by through the administration of anti-histamines, proton pump inhibitors, and antacids [7]. The etiology of gastritis remains unclear and is thought to be associated with the imbalance between protective and 
destructive factors. Cytokines, such as IL-8 and TNF-a, play important roles in the inflammatory reactions involved in the progression of gastritis [8]. Overexpression and translocation of nuclear factor kappa $B$ (NF-KB) subunits to the nucleus increase the expression of proinflammatory mediators such as TNF-a [9]. Inhibition of IL-8 secretion in TNF-a treated human gastric epithelial cells by suppressing NF-KB signaling may exert anti-inflammatory effects [10]. Studies have shown that the inhibition of NF-KB activity may reduce the severity of inflammation [11]. Among the drugs used for the treatment of gastritis, antacids (including omeprazole; $\mathrm{OME}$ ) are the most common agents that alleviate the associated symptoms. However, these drugs may exert adverse effects such as dry mouth, nausea, bloating, constipation, insomnia and peripheral neuritis [12]. Therefore, alternative and complementary remedies that may exhibit anti-inflammatory effects and regenerate stomach tissues without adverse effects have received global attention.

Emerging evidence indicates that some flavonoid compounds, such as baicalin and baicalein, exert antiinflammatory effects. Baicalin belongs to flavonoid compounds, respectively. Baicalein, baicalin, and wogonin could exert an anti-inflammatory effect on LPS-mediated vascular inflammatory response by inhibiting the MAPK and NF-KB pathways [13]. Baicalin and baicalein promote health by preventing $H$. pylori infection, which interferes with $H$. pylori growth and virulence [14]. Given that, antioxidant and antiinflammatory natural molecules may be potential candidates for gastritis therapy, most of all, they performed the characteristics of efficacy and safety. Baicalin is a flavonoid with good anti-inflammatory, antioxidant properties, and antimicrobial activities, which is a great potential in preventing and inhibiting tumors [15]. Therefore, baicalin is one kind of active components with the important synergies, they have been widely used in China due to their crucial pharmacological effects, including enhancing immune function and anti-inflammatory [16]. Here, we employed a Tandem Mass Tag (TMT) labeling system, a high throughput, efficiency, and sensitivity tool, to analyze the expression of various proteins in the rat gastric antrum following ethanol-induced gastritis. Proteomics technology may uncover the molecular machinery underlying signaling pathways [17].

In this study, we used quantitative proteomics to elucidate the mechanism of baicalin to explain the differences in outcomes of ethanol-induced chronic gastritis. Furthermore, we focused on the differentially expressed proteins (DEPs) and signaling pathways, which may reveal potential targets for the development of drugs for the effective treatment of chronic gastritis.

\section{Methods}

\section{Reagents and materials}

Baicalin were obtained from Yuanye Biological; Omeprazole (Luoxin, China); IL-2, IL-8, and TNF-a were obtained from Sinobest Biotech (Shanghai, China); TPM2, GIMAP4, Bcl-2, and Mpc1 were supplied by Biosynthesis (Beijing, China); EGF was provided by Affinity (USA) and Tandem Mass Tags (TMTs) ${ }^{10}$ were purchased from (Thermo Fisher, USA); Tris (2-carboxyethyl) phosphine (TCEP) was obtained from Thermo Fisher Scientific (USA) and ACQUITY UPLC BEH C18 column was procured from Waters (USA). 


\section{Animal experiments and sample preparation}

Male Sprague-Dawley (SD) rats, weighing $180 \pm 20 \mathrm{~g}$, were obtained from the Animal Experiment Center of Shanghai University of Traditional Chinese Medicine, Shanghai, China. The animals were housed in a controlled environment $\left(12 \mathrm{~h}\right.$ light and $25^{\circ} \mathrm{C}$ ), and were given free access to food and water. All experimental procedures were carried out in accordance with the Chinese Guidelines for the Care and Use of Laboratory Animals (Permit No. SZY201712009). Thirty-two rats were randomly assigned to four groups as follows ( $n=8)$ : normal group, model group, BCL group ( $50 \mathrm{mg} / \mathrm{kg}$ of baicalin), and OME group (20 mg/kg of OME; positive group). Gastritis rat model was induced with $56 \%$ ethanol according to an established procedure [18]. After $12 \mathrm{~h}$ fasting, the rats received $56 \%$ ethanol $(8 \mathrm{~g} / \mathrm{kg})$ through gastrogavage twice a week (every Tuesday and Friday). After 4 weeks, the chronic gastritis model was fully established. In the model therapy groups, BCL, or OME were orally administered. The normal and model groups received saline following the same protocol. All the therapeutics were administered via gastrogavage once daily for 7 days. On the eighth day, all rats were anaesthetized by $2 \%$ pentobarbital sodium $(0.3 \mathrm{ml} / 100 \mathrm{~g})$ via intraperitoneal injection and blood was taken from the abdominal aorta, then the stomach was immediately excised.

\section{Histopathological analysis}

The stomach tissue was carefully cut along the large curvature, cleaned with saline and blotted with filter paper. Tissue samples close to the gastric antrum were obtained from each group. The gastric antrum tissues were fixed in a $4 \%$ paraformaldehyde for $24 \mathrm{~h}$, and sectioned and embedded in paraffin. Sections (5 mm thickness) were deparaffinized, stained with hematoxylin and eosin (H\&E), and examined under a light microscope.

\section{Determination of IL-2, IL-8, and TNF-a levels in serum}

Plasma was separated from $4 \mathrm{~mL}$ of blood by centrifugation at $4^{\circ} \mathrm{C}$ for $15 \mathrm{~min}$ at $3000 \times g$. Serum levels of IL-2, IL-8 and TNF-a were measured with enzyme-linked immunosorbent assays (ELISAs) according to the manufacturer's instructions.

\section{Protein isolation, digestion, and labeling with TMT reagent}

Tissue samples $(5 \times 5 \mathrm{~mm})$ close to the gastric antrum were collected in all groups, ground into a powder in liquid nitrogen, and extracted with lysis buffer in a ratio of 1:12 (8 M urea, 1\% sodium dodecyl sulfate [SDS], including protease inhibitors). These samples were chilled on ice for $30 \mathrm{~min}$ and finally lysed with sonication on ice for $3 \mathrm{~min}$. These homogenized samples were centrifuged for $20 \mathrm{~min}$ at $12,000 \times g$ to obtain protein containing supernatants. TCEP was added to each sample (each representing $100 \mu \mathrm{g}$ of protein) and left to react for 60 min before iodoacetamide was added. The solution was incubated at $20^{\circ} \mathrm{C}$ in the dark for $40 \mathrm{~min}$. Finally, acetone was added to each tube for precipitation. The precipitate was collected and dissolved in TEAB. Trypsin was added to the samples at a ratio of 1:50 (enzyme: protein) 
and the samples were incubated at $37^{\circ} \mathrm{C}$ overnight. Tandem mass tags $\mathrm{TMT}^{10}$ of different molecular weights (126 to $131 \mathrm{Da}$ ) served as isobaric tags for relative quantification.

\section{Liquid chromatography with tandem mass spectrometry (MS/MS) analysis}

The peptides were subjected to elution on Acquity UPLC BEH C18 column in an ultra high-pressure liquid chromatography (UPLC) system (Waters, USA) with a mobile phase of 2-80\% acetonitrile, using ammonia to $\mathrm{pH} 10$. The flow rate was $200 \mu \mathrm{L} / \mathrm{min}$. The peptides were subjected to nanoelectrospray ionization and followed by MS/MS analysis on Q-Exactive instrument (Thermo, USA) coupled to an HPLC system (EASYnLC1200) with a reverse-phase C18 column. For MS scans, the $m / z$ scan range was 350-1,300 Da.

\section{Bioinformatics and statistical analysis}

MS/MS spectra were collected from raw data and converted into an MGF format in Proteome Discoverer ${ }^{\mathrm{TM}}$ Software 2.1 (containing 29,969 reference protein sequences). The parameters of the protein database were as follows: Max.Missed Cleavage Sites were 2, Precursor Mass Tolerance was 20 ppm, Fragment Mass Tolerance was $0.02 \mathrm{Da}$, validation was based on p-values and the peptide filtration parameter was false discovery rate $(F D R) \leq 0.01$. By means of the $G O$ database, genes and proteins were classified in accordance with their participation in biological processes, cellular components and molecular function (http://www.geneontology.org/). In the KEGG database

(http://www.genome.ad.jp/kegg/pathway.html), the DEPs involved in various signaling pathways and biological metabolic processes were identified. The DEPs and the predicted PPI network were analyzed with STRING database (https://string-db.org/) [19]. The proteins we identified had to satisfy the criteria fold change $(\mathrm{FC})<0.83$ or $>1.20$ and $P<0.05$ to be considered DEPs. All results were reported as mean \pm S.D. The data were evaluated using the SPSS 16.0 software. The significance level was set to $P<0.05$.

\section{Western Blot analysis of DEPs}

The total protein extract of the gastric antrum samples corresponding to $20 \mu \mathrm{g}$ of proteins from each group was resolved by SDS-polyacrylamide gel electrophoresis using $10 \%$ gel and then transferred to PVDF membranes, blocked with 5\% non-fat dried-milk in TBST buffer for $1 \mathrm{~h}$, and incubated with antibodies (1:1000) against TPM2, GIMAP4, Mpc1, EGF, Bcl-2 and $\beta$-actin at $4^{\circ} \mathrm{C}$ overnight. The membranes were subsequently incubated with a secondary antibody (anti-lgG) at 1:2000 dilution for $1 \mathrm{~h}$ at room temperature and the membrane was washed thrice. Finally, the antibody-antigen complexes and protein bands were quantified by densitometry using a Quantitative Gel and Western Imaging System (FluorChem Q).

\section{Results}

Protective effects of baicalin against ethanol-induced chronic gastritis in rat 
After administration of ethanol to the tissues in the model group, extensive lymphocyte infiltration was observed in the intrinsic, submucosal, and serosal layer. Edema and congestion were apparent in the submucosal and intrinsic layers. Lymphocyte infiltration and edema reduced in $B C L$ group as compared with model group, the symptoms of gastritis were significantly alleviated in BCL group (Fig.1A). The levels of IL-2, IL-8, and TNF-a significantly increased $(P<0.01)$ in model group. BCL treatment may downregulate the expression of IL-2, IL-8 and TNF-a (Fig. 1B). Meanwhile, EGF and Bcl-2 were significantly upregulated following BCL treatment (Fig. 1C). These findings demonstrated that baicalin exerted therapeutic effects on ethanol-induced chronic gastritis in rats.

\section{TMT-based quantification of the gastric antrum proteomes}

The proteome was performed on the gastric antrum divided into four groups based on different treatments. Our proteomic workflow was shown in Fig.2A. In total, 4,452 proteins were identified from 20,842 peptides with high confidence. A cutoff value of 1.2 -fold change and $P$-values $<0.05$ were used in our study $[20,21]$. According to the matching degree of the peptides, it was found that the DeltaM [ppm] of most peptides is concentrated in -10 to 10 (Fig.2B). Compared to the control group, 318 DEPs were identified, of which 216 and 102 were up- and down-regulated respectively in ethanol-treated rats. Furthermore, compared with the model group, there were 107 DEPs in baicalin, of which 44 and 63 were up- and down-regulated, respectively (Fig.2C).

\section{Biological function analysis}

To examine the biological functions of DEPs in the therapeutic effect of $B C L$ on chronic gastritis, DEPs were categorized according to their associated biological processes, molecular function and cell localization with the Gene Ontology (GO). In the biological processes category, DEPs were significantly enriched in cell killing, immune system process, and multicellular organismal process. In the cellular component category, these DEPs were involved in cell part, extracellular region, and extracellular region part. In the molecular function category, the DEPs were significantly enriched in binding, catalytic activity and molecular transducer activity. Thus, BCL-affected DEPs may play important regulatory roles in fundamental biological processes, immune and binding during BCL treating chronic gastritis.

KEGG pathway enrichment analysis demonstrated that BCL-affected signaling pathways were significantly enriched in complement and coagulation cascades, PI3K-Akt and NF-KB. Interestingly, several common pathways including MAPK signaling pathway, such as Col4a2 and HSP27, which were strongly associated with BCL treatment (Fig.3A).

\section{Protein interaction networks of BCL-affected DEPs}

STRING was carried out to analyze the interactions between DEPs that showed functional interactions [22]. A protein-protein interaction (PPI) network may integrate and analyze the known PPIs via proteomic and gene data. $44 \mathrm{Up}$ - and 63 down-regulated proteins and constructed PPI networks were used to study the relations between chronic gastritis and BCL treatment. In the network, Acta, Prothrmbin, 
Fibrinogen beta chain, and TPM2 were associated with other DEPs in PPI. Interestingly, BCL-affected DEPs were classified into immune process, regulation of actin cytoskeleton and inflammation process, which formed the large network associated with gastritis (Fig.3B).

\section{Western Blot confirmation}

From the data of proteomics, the expression level of some proteins performed significant change between BCL and model groups. From the results of Western Blot (Fig. 4), compared to model group, it was confirmed that the expression of ICAM-1 and GIMAP4 were reduced in baicalin group. On the contrary, TPM2 and Mpc1 expression increased in baicalin group. These results demonstrated that the expression trend of these proteins in tissue samples was consistent with our proteomics findings and confirmed the reliability of the proteomics data.

\section{Discussion}

A previous study has illustrated the therapeutic effects of baicalin on ethanol-induced gastritis through a reduction in the generation of inflammatory cytokines. It have demonstrated that the intake of ethanol causes acute or chronic gastritis [23]. The molecular structure of ethanol includes both hydrophobic alkyls and hydrophilic hydroxyls that may attenuate the gastric mucosal barrier defense system and reduce the ability of the gastric mucosa to defend against gastric acid invasion, leading to mucosal edema, erosion, hemorrhage, and necrosis [24]. Simultaneously, EGF receptor ligands have been implicated in the induction of gastric cell proliferation and function [25]. Baicalin significantly upregulated the expression of EGF and protected the damaged gastric mucosa. Among various the regulatory factors involved in apoptosis, $\mathrm{Bcl}-2$ regulation is particularly important, because reduction in Bcl-2 level causes apoptosis-mediated cell death [26, 27].

Quantitative proteomics technology is an effective tool to study the mechanisms underlying natural compounds-mediated gastritis alleviation. Proteomic technology may identify novel potential diagnostic biomarkers in the gastrointestinal stroma [28]. Previous study demonstrated that TPM2 may be involved in cancer formation. Beta-tropomyosin ( $\beta$-tropomyosin, TPM2) gene is known to contribute to series of rare myopathies including many kinds of diseases, and is crucial for the regulation of muscle contraction associated with actin and the troponin complex [29]. It was also essential for the stability of actin thin filaments, as well as many functions of cell processes such as migration. Report showed that TPM2 was down-regulated in colorectal adenomas and cancers as compared to normal colon tissues [30]. Several early studies also demonstrated that TPM2 was down-regulated in human esophageal squamous cell carcinoma, while unregulated in ovarian and breast cancer [31-33]. Form the validation analysis of Western Blot, BCL could up-regulate the expression level of TPM2, which was consistent with results of proteomics. From the results of TMT proteomics, TPM2 was down-regulated in model group, while upregulated in BCL group, TPM2 may take part in the gastritis formation, so we speculated it may be a biomarker candidate for ethanol-gastritis. Additionally, GO analysis demonstrated that TPM2 was involved in molecular function. 
GIMAP4 is reported to exhibit GTPase activity and function in cellular transport processes. GIMAP4 may affect the levels of IFN- $\gamma$ and is involved in the IFN- $\gamma$ signaling pathway in Th1 cells [34]. Mpc1 takes part in the control of rate-limiting pyruvate transport through the inner mitochondrial membrane [35]. Mpc1 overexpression inhibits the proliferation, migration, and invasiveness of gastric cancer cells [36].

Therefore, we can speculate that TPM2, GIMAP4, and Mpc1 are potential targets of baicalin in treating chronic gastritis. Baicalin affected several signaling pathways, including PI3K-Akt, NF-KB, and MAPK signaling pathways. The underlying mechanism involves anti-inflammatory, anti-apoptotic effects, and induction of endogenous factors for cytoprotection.

\section{Conclusions}

In this study, a total of 107 DEPs were identified from the BCL group, when compared to the ethanolinduced gastritis group, the results showed for the first time that quantitatively evaluating the gastric antrum proteins by TMT in ethanol-induced gastritis and BCL-treated gastritis rats performs a feasible and effective strategy for identify gastritis associated with proteins, pathways, networks, and potential therapeutic targets. This study may also demonstrate that baicalin played a crucial role in treating ethanol-induced gastritis. Consequently, the present study widens the range of treatment strategies for chronic gastritis and highlights potential drug targets for modulation of inflammation and apoptosis.

\section{Abbreviations}

TMT: Tandem mass spectrometry tag; BCL: Baicalin; IL-2: Interleukin-2; TNF-a: Tumor necrosis factor-a; EGF: Epidermal growth factor; Bcl-2: B-cell lymphoma-2; TPM2: Tropomyosin beta chain 2; GIMAP4: GTPase IMAP family member 4; Mpc1: Mitochondrial pyruvate carrier 1.

\section{Declarations}

\section{Acknowledgements}

We thank the Technique Support, Shanghai Majorbio Bio-pharm Technology Co., Ltd.

\section{Author Contributions}

Wan-li Ji: Data curation, Formal analysis, Methodology, Visualization, Writing- Original draft preparation. Yan Huo: Data curation, Methodology, Validation; Yu Zhang: Methodology, Validation; Xin-hong Wang: Conceptualization, Writing-review and editing. Yi-fan Zhang: Conceptualization, Methodology, Writingreview and editing, Supervision, Funding acquisition.

\section{Funding}

This work was supported by the National Natural Science Foundation of China (grant No. 81774183), and sponsored by Scientific Research Foundation of Shanghai University of Medicine and Health Sciences 
(grant number E3-0200-21-201011-34).

\section{Availability of data and materials}

The MS proteomics raw data have been deposited in the iProX system (http://www.iprox.org/index) under the identifier IPX0001246000.

\section{Ethics approval and consent to participate}

All experimental procedures were carried out in accordance with the Chinese Guidelines for the Care and Use of Laboratory Animals (Permit No. SZY201712009).

\section{Consent for publication}

Not applicable.

\section{Competing interests}

There are no competing interests.

\section{Author details}

${ }^{1}$ School of Pharmacy, Shanghai University of Medicine and Health Sciences, Shanghai 201318, China

${ }^{2}$ School of Pharmacy, Shanghai University of Traditional Chinese Medicine, Shanghai, 201203, China

${ }^{3}$ Yueyang Hospital of Integrated Traditional Chinese and Western Medicine, Shanghai University of Traditional Chinese Medicine, Shanghai, 200437, China

\section{References}

1. Diaz-Rivas JO, Herrera-Carrera E, Gallegos-Infante JA, Rocha-Guzman NE, Gonzalez-Laredo RF, Moreno-Jimenez MR, Ramos-Gomez M, Reynoso-Camacho R, Larrosa-Perez M, Gallegos-Corona MA: Gastroprotective potential of Buddleja scordioides Kunth Scrophulariaceae infusions; effects into the modulation of antioxidant enzymes and inflammation markers in an in vivo model. Journal of ethnopharmacology 2015, 169:280-286.

2. Federico A, Cotticelli G, Festi D, Schiumerini R, Addolorato G, Ferrulli A, Merli M, Lucidi C, Milani S, Panella $\mathrm{C}$ et al: The effects of alcohol on gastrointestinal tract, liver and pancreas: evidence-based suggestions for clinical management. European review for medical and pharmacological sciences 2015, 19(10):1922-1940.

3. Lieber CS: Gastric ethanol metabolism and gastritis: Interactions with other drugs, Helicobacter pylori, and antibiotic therapy (1957-1997) - a review. Alcoholism-Clinical and Experimental Research 1997, 21(8):1360-1366. 
4. Bode C, Bode JC: Alcohol's role in gastrointestinal tract disorders. Alcohol health and research world 1997, 21(1):76-83.

5. Liu Y, Tian X, Gou L, Fu X, Li S, Lan N, Yin X: Protective effect of I-citrulline against ethanol-induced gastric ulcer in rats. Environ Toxicol Pharmacol 2012, 34(2):280-287.

6. del Valle JC, Salvatella M, Rossi I, Andrade R, Gutierrez Y, Pereda C, Samper B, Feliu JE: Impairment of $\mathrm{H}+-\mathrm{K}+-\mathrm{ATP}$ ase-dependent proton transport and inhibition of gastric acid secretion by ethanol. $\mathrm{Am} \mathrm{J}$ Physiol Gastrointest Liver Physiol 2001, 280(6):G1331-1340.

7. Laloo D, Prasad SK, Krishnamurthy S, Hemalatha S: Gastroprotective activity of ethanolic root extract of Potentilla fulgens Wall. ex Hook. Journal of ethnopharmacology 2013, 146(2):505-514.

8. Kang GD, Kim DH: Ponciretin attenuates ethanol-induced gastric damage in mice by inhibiting inflammatory responses. International immunopharmacology 2017, 43:179-186.

9. Shi H, Ma J, Mi C, Li J, Wang F, Lee JJ, Jin X: Amorfrutin A inhibits TNF-alpha-induced NF-kappaB activation and NF-kappaB-regulated target gene products. Int Immunopharmacol 2014, 21(1):56-62.

10. Fumagalli M, Sangiovanni E, Vrhovsek U, Piazza S, Colombo E, Gasperotti M, Mattivi F, De Fabiani E, Dell'Agli M: Strawberry tannins inhibit IL-8 secretion in a cell model of gastric inflammation. Pharmacol Res 2016, 111:703-712.

11. Isomoto H, Mizuta Y, Miyazaki M, Takeshima F, Omagari K, Murase K, Nishiyama T, Inoue K, Murata I, Kohno S: Implication of NF-kappa B in Helicobacter pylori-associated gastritis. American Journal of Gastroenterology 2000, 95(10):2768-2776.

12. Matheson AJ, Jarvis B: Lansoprazole: an update of its place in the management of acid-related disorders. Drugs 2001, 61(12):1801-1833.

13. Ku SK, Bae JS: Baicalin, baicalein and wogonin inhibits high glucose-induced vascular inflammation in vitro and in vivo. BMB reports 2015, 48(9):519-524.

14. Chen ME, Su CH, Yang JS, Lu CC, Hou YC, Wu JB, Hsu YM: Baicalin, Baicalein, and Lactobacillus Rhamnosus JB3 Alleviated Helicobacter pylori Infections in Vitro and in Vivo. 2018, 83(12):3118-3125.

15. Wu Y, Wang F, Fan L, Zhang W, Wang T, Du Y, Bai X: Baicalin alleviates atherosclerosis by relieving oxidative stress and inflammatory responses via inactivating the NF-kappaB and p38 MAPK signaling pathways. Biomedicine \& pharmacotherapy = Biomedecine \& pharmacotherapie 2018, 97:1673-1679.

16. Mir-Palomo S, Nacher A, Ofelia Vila Buso MA, Caddeo C, Manca ML, Manconi M, Diez-Sales O: Baicalin and berberine ultradeformable vesicles as potential adjuvant in vitiligo therapy. Colloids and surfaces B, Biointerfaces 2019, 175:654-662. 
17. Yagüe VHGPCBJAJ: Changes in protein expression in developed experimental pancreatitis: a Tandem Mass Tag (TMT) proteomics analysis. Pancreatology 201717 (5):S7-S8.

18. Vazquez-Ramirez R, Olguin-Martinez M, Kubli-Garfias C, Hernandez-Munoz R: Reversing gastric mucosal alterations during ethanol-induced chronic gastritis in rats by oral administration of Opuntia ficus-indica mucilage. World journal of gastroenterology 2006, 12(27):4318-4324.

19. Zhang X, Chen Y, Pan J, Liu X, Chen H, Zhou X, Yuan Z, Wang X, Mo D: iTRAQ-based quantitative proteomic analysis reveals the distinct early embryo myofiber type characteristics involved in landrace and miniature pig. BMC genomics 2016, 17:137.

20. Wang J, Yan S, Zhang W, Zhang H, Dai J: Integrated proteomic and miRNA transcriptional analysis reveals the hepatotoxicity mechanism of PFNA exposure in mice. Journal of proteome research 2015, 14(1):330-341.

21. Prado RS, Bailao AM, Silva LC, de Oliveira CM, Marques MF, Silva LP, Silveira-Lacerda EP, Lima AP, Soares CM, Pereira M: Proteomic profile response of Paracoccidioides lutzii to the antifungal argentilactone. Frontiers in microbiology 2015, 6:616.

22. Woo J, Han D, Wang JI, Park J, Kim H, Kim Y: Quantitative Proteomics Reveals Temporal Proteomic Changes in Signaling Pathways during BV2 Mouse Microglial Cell Activation. Journal of proteome research 2017, 16(9):3419-3432.

23. Park SW, Oh TY, Kim YS, Sim H, Park SJ, Jang EJ, Park JS, Baik HW, Hahm KB: Artemisia asiatica extracts protect against ethanol-induced injury in gastric mucosa of rats. Journal of gastroenterology and hepatology 2008, 23(6):976-984.

24. Ning JW, Lin GB, Ji F, Xu J, Sharify N: Preventive effects of geranylgeranylacetone on rat ethanolinduced gastritis. World journal of gastroenterology 2012, 18(18):2262-2269.

25. Franic TV, Judd LM, Nguyen NV, Samuelson LC, Loveland KL, Giraud AS, Gleeson PA, van Driel IR: Growth factors associated with gastric mucosal hypertrophy in autoimmune gastritis. Am J Physiol Gastrointest Liver Physiol 2004, 287(4):G910-918.

26. Moya A, Sakamaki K, Mason BM, Huisman L, Foret S, Weiss Y, Bull TE, Tomii K, Imai K, Hayward DC et al: Functional conservation of the apoptotic machinery from coral to man: the diverse and complex Bcl-2 and caspase repertoires of Acropora millepora. BMC genomics 2016, 17:62.

27. Lu QL, Abel P, Foster CS, Lalani EN: bcl-2: role in epithelial differentiation and oncogenesis. Human pathology 1996, 27(2):102-110.

28. Atay S, Wilkey DW, Milhem M, Merchant M, Godwin AK: Insights into the Proteome of Gastrointestinal Stromal Tumors-Derived Exosomes Reveals New Potential Diagnostic Biomarkers. Molecular \& cellular proteomics : MCP 2018, 17(3):495-515. 
29. Mroczek M, Kabzinska D: A novel TPM2 gene splice-site mutation causes severe congenital myopathy with arthrogryposis and dysmorphic features. 2017, 58(2):199-203.

30. Luo YX, Cui J, Wang L, Chen DK, Peng JS, Lan P, Huang MJ, Huang YH, Cai SR, Hu KH et al: Identification of cancer-associated proteins by proteomics and downregulation of beta-tropomyosin expression in colorectal adenoma and cancer. Proteomics Clinical applications 2009, 3(12):1397-1406.

31. Zhang J, Wang K, Zhang J, Liu SS, Dai L, Zhang JY: Using proteomic approach to identify tumorassociated proteins as biomarkers in human esophageal squamous cell carcinoma. Journal of proteome research 2011, 10(6):2863-2872.

32. Tang HY, Beer LA, Tanyi JL, Zhang R, Liu Q, Speicher DW: Protein isoform-specific validation defines multiple chloride intracellular channel and tropomyosin isoforms as serological biomarkers of ovarian cancer. Journal of proteomics 2013, 89:165-178.

33. Li DQ, Wang L, Fei F, Hou YF, Luo JM, Zeng R, Wu J, Lu JS, Di GH, Ou ZL et al: Identification of breast cancer metastasis-associated proteins in an isogenic tumor metastasis model using two-dimensional gel electrophoresis and liquid chromatography-ion trap-mass spectrometry. Proteomics 2006, 6(11):33523368.

34. Heinonen MT, Kanduri K, Lahdesmaki HJ, Lahesmaa R, Henttinen TA: Tubulin- and actin-associating GIMAP4 is required for IFN-gamma secretion during Th cell differentiation. Immunology and cell biology 2015, 93(2):158-166.

35. Bricker DK, Taylor EB, Schell JC, Orsak T, Boutron A, Chen YC, Cox JE, Cardon CM, Van Vranken JG, Dephoure $\mathrm{N}$ et al: A mitochondrial pyruvate carrier required for pyruvate uptake in yeast, Drosophila, and humans. Science (New York, NY) 2012, 337(6090):96-100.

36. Zhou X, Xiong ZJ, Xiao SM, Zhou J, Ding Z, Tang LC, Chen XD, Xu R, Zhao P: Overexpression of MPC1 inhibits the proliferation, migration, invasion, and stem cell-like properties of gastric cancer cells. OncoTargets and therapy 2017, 10:5151-5163.

\section{Figures}




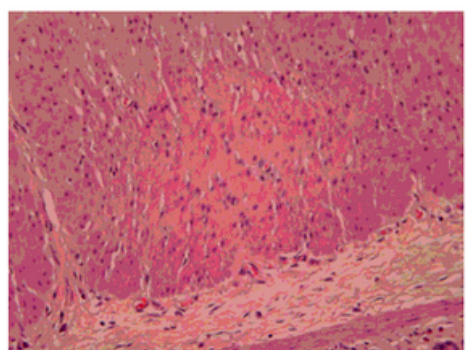

Normal

B

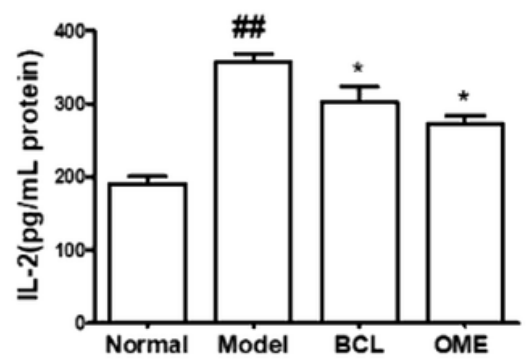

Model

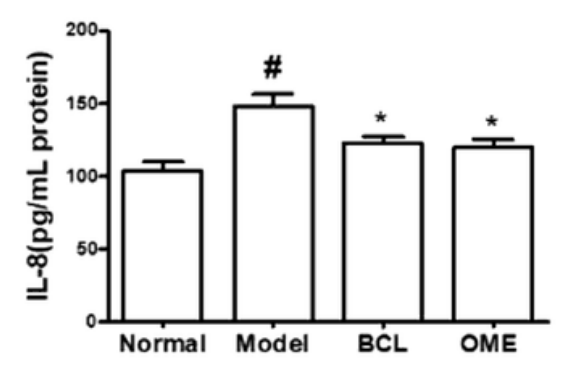

BCL

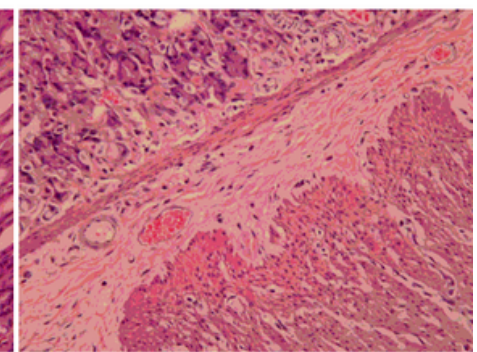

OME

C
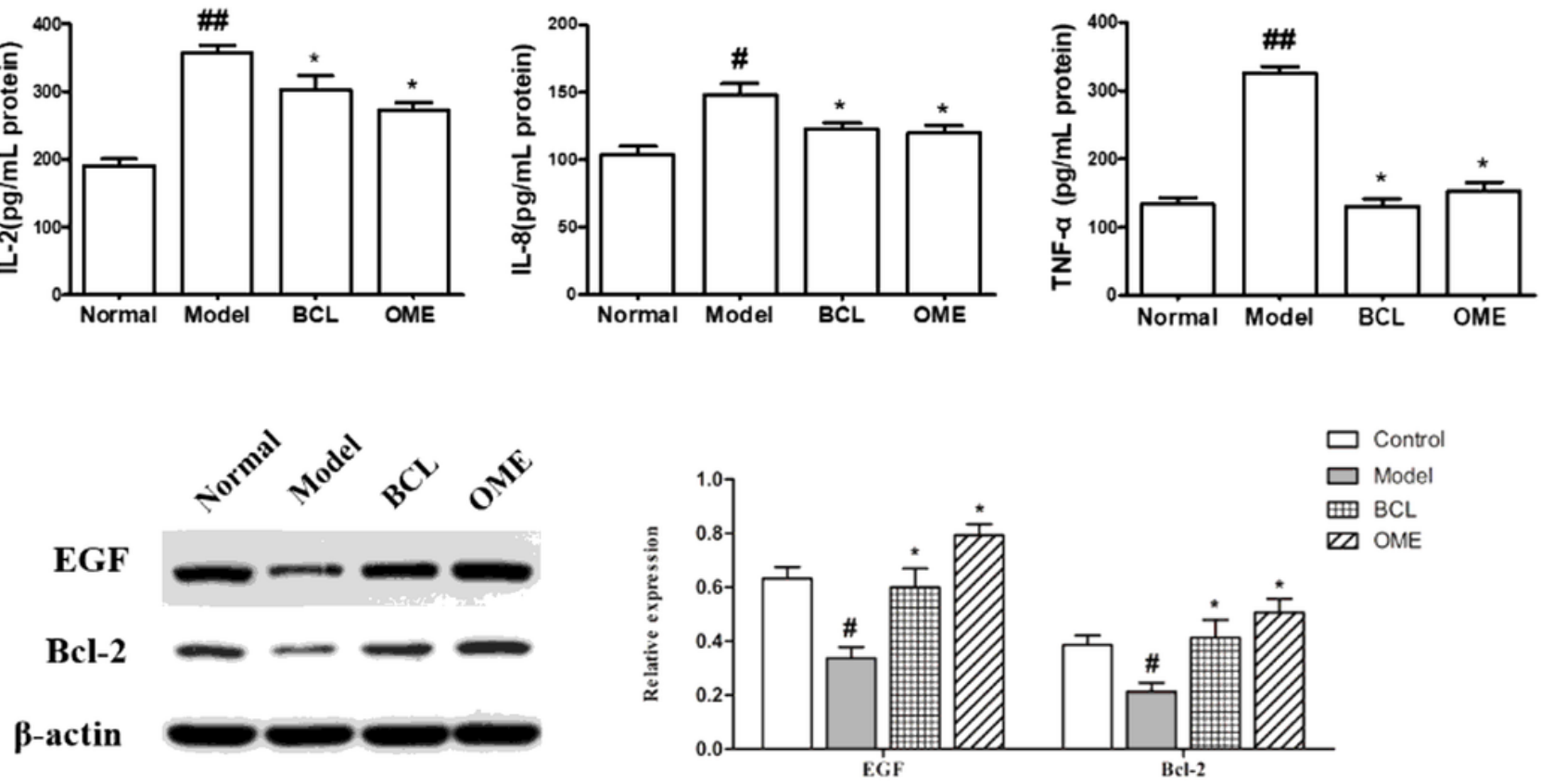

Figure 1

Effects of baicalin on chronic gastritis in rats. A: H\&E staining (magnification 200x); B: Determination of IL-2, IL-8 and TNF-a levels in serum and cytoprotective factors; C: Western Blot analysis of EGF and Bcl-2, $\# P<0.05$ vs. Normal group, ${ }^{*}<0.05$ vs. Model group. 
A
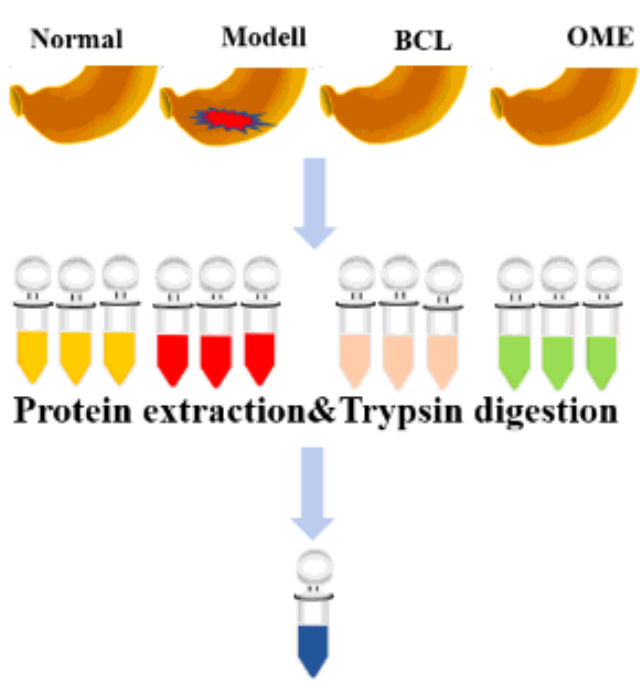

TMT labeling peptides

\section{LC-MS/MS}
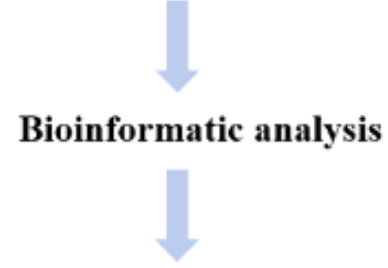

\section{Validation}

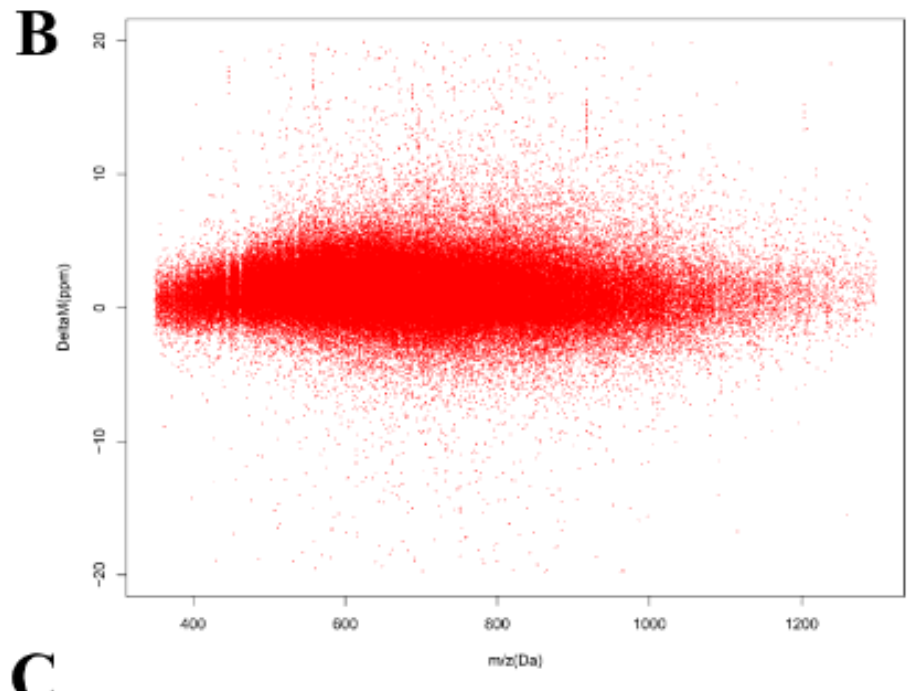

C

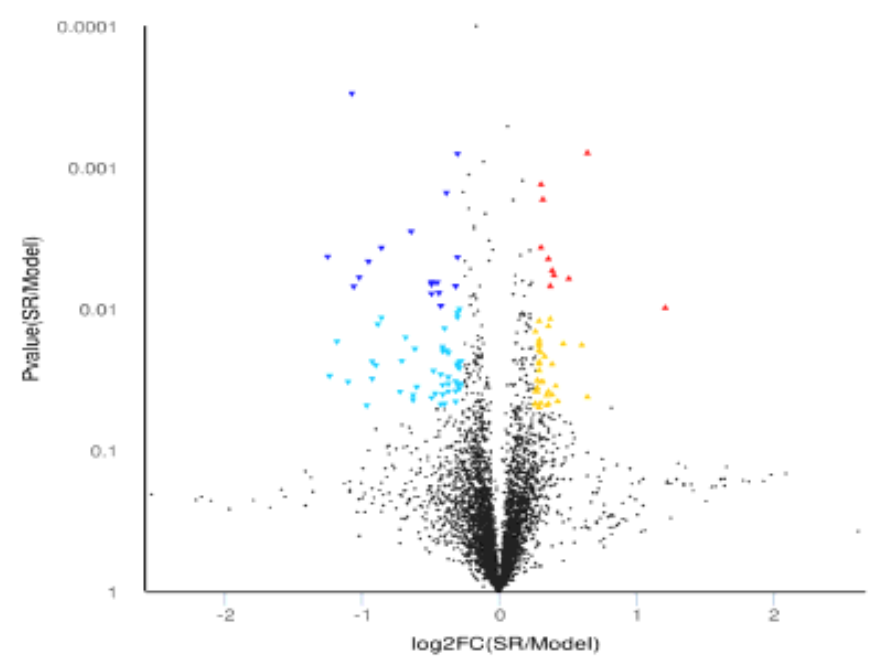

down-p-0.01

down-p-0.05

nosig

up-p-0.05

up-p-0.01

\section{Figure 2}

TMT quantitative proteomic workflow and visual analysis. A: TMT proteomic workflow; B: Gastric tissue protein peptides error; C: Volcano plot of DEPs in BCL group compared with model group. The $\mathrm{X}$ axis was log2-fold change of high and low expression proteins in BCL vs model. The $Y$ axis represents the false discovery (FDR) of the change fold. Different colors represent different FDR values. 


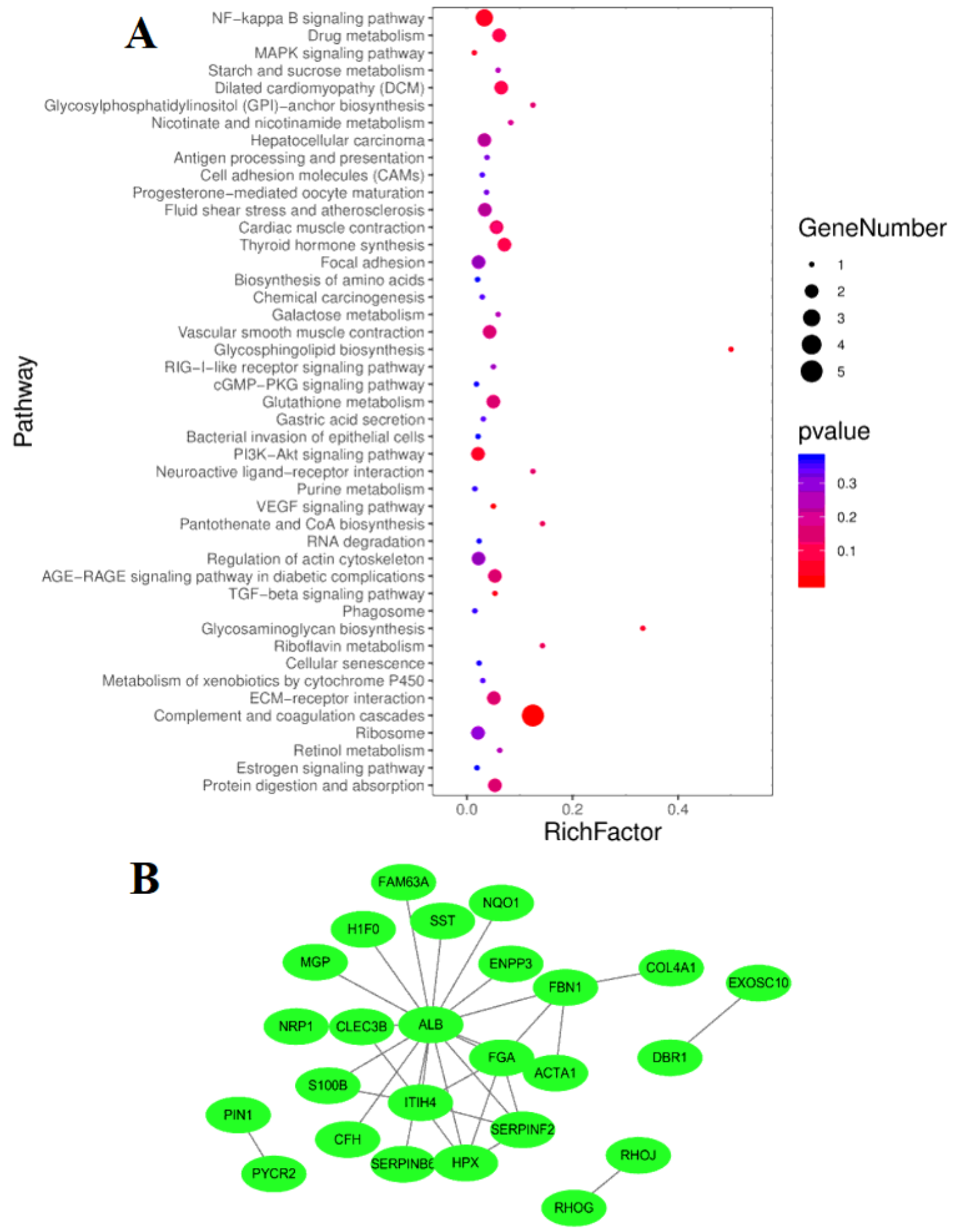

\section{Figure 3}

The KEGG and PPI analysis of BCL-affected DEPs. A: The KEGG analysis, the size of the circle represents the amount of protein enrichment, colour represents the significance of enrichment, and the right-hand colour gradient represents P value. B: The PPI analysis of BCL-affected DEPs, 25 DEPs out of 107 DEPs were involved in the PPI networks, which contained 25 nodes and 30 edges. 
A

Normal Model BCL OME

TPM2

Mpc1

\section{GIMAP4}

ICAM-1

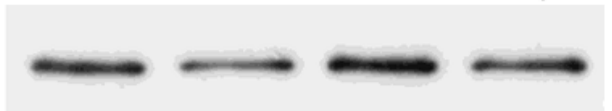

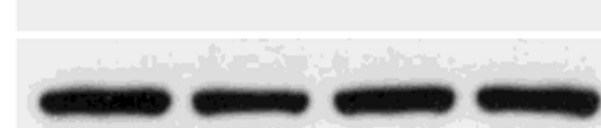

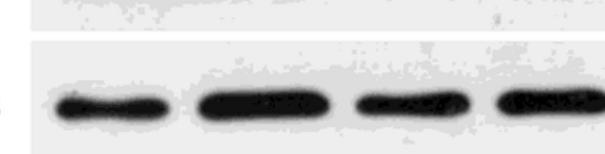

ICAM-1

$\beta$-actin

\section{B}
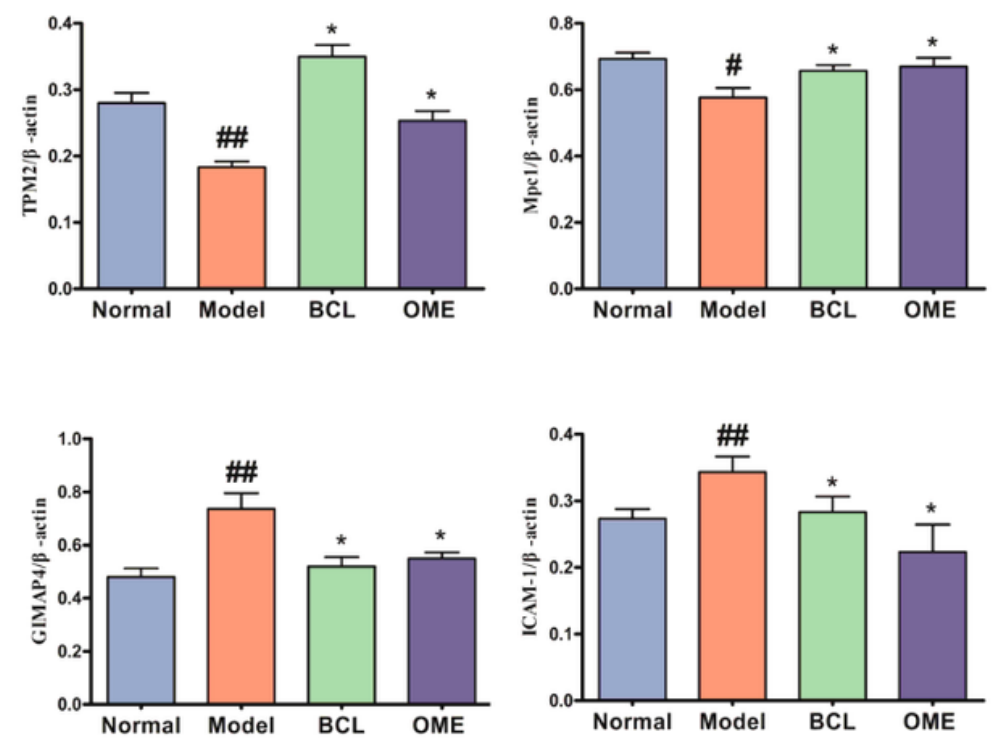

Figure 4

DEPs-affected by BXD and Western Blot validation of DEPs. A: In rats subjected to BCL treatment, the expression levels of GIMAP4 and ICAM-1 decreased as compared with the rat from the model group, while the expression levels of TPM2 and Mpc1 were increased by BCL. B: Expression levels of the DEPs by western blot was normalized to $\beta$-action expression. The relative intensity of bar chart demonstrated quantification of proteins, the bars showed means \pm SEM of three independent experiments. \#\#P $<0.01$ vs. Control group, $\# \mathrm{P}<0.05$ vs. Control group, ${ }^{*} \mathrm{P}<0.05$ vs. Model group. 